\title{
EDITORIAL
}

\section{Advanced Analyses of Technology Innovations, Patents, and Intellectual Property}

\section{Amy J. C. Trappey}

Distinguished Professor

Department of Industrial Engineering and Engineering Management, National Tsing Hua University, Taiwan trappey@ie.nthu.edu.tw

DOI: 10.5875 /ausmt.v3i3.252

(Published on line 1 September 2013)

A good indicator of the technical competitiveness of a country or an enterprise is the number of high quality international patents it owns. The latest statistics from the World Intellectual Property Organization (WIPO) showed that USA, Japan, Germany, China, and South Korea are among the top patent filing countries [1]. WIPO data indicate that these countries and their global companies are directing significant resources toward engineering innovations, design inventions, and technology R\&D. The end result will be to capitalize their intellectual assets for competitive advantage.

The huge and ever growing data and knowledge content of international patent (cyber-) depositories creates great challenges for engineers, researchers, and technology strategists to fully utilize the wealth of knowledge [2]. Most critically, engineers must make correct R\&D decisions based on the complex sets of patent information. This special issue features research papers focusing on the advances of representing, modeling, reasoning, analyzing, and synthesizing knowledge related to innovations, intellectual property (IP), patents, and technology adoptions. For industries with short life cycles and technical products (e.g., semiconductor, LED, and consumer electronics), state-of-the-art knowledge engineering approaches for IP and patent analysis are essential for sustaining competitive advantage. We have accepted five articles in this special issue and each has developed methodologies using industrial cases for verification. The papers are highlighted briefly as follows.

The article "Applying Hybrid-Quantity Analysis in the Asia Semiconductor Industry," co-authored by Chin-yuan Fan, Ming-Fong Lai, and Shu-Hao Chang, provides an integrated approach to explore competing technological and strategic-group relationships within the semiconductor industry in the Asia-Pacific region. They use a combination of patents, multidimensional scaling, and K-means clustering to conduct their hybrid analyses. Their findings provide recommendations for technology development strategies.

The paper, entitled "Ontology-based Patent Licensing and Litigation Strategic Knowledge System for the Light Emitting Diode Industry" co-authored by Amy J.C. Trappey, Yu-Hui Wang, Charles V. Trappey, Chun-Yi $\mathrm{Wu}$, and Tzu-Hsuan Lin, develops a scientific and analytical approach for strategic cross-licensing decision support, particularly for the extremely competitive global companies in LED industry. 
Yu-Hui Wang and Benjamin Liu present their research in the paper entitled "Innovation Effect on Patent Pool Formation: Empirical Case of Philips' Patents in Digital Versatile Disc 3C." The paper evaluates both the quantity and quality of Philips' patents in a leading DVD patent pool. The legitimacy of patent pools and their strategic value are discussed and investigated using the DVD 3 C patent pool as the case example.

In the paper "Application of the Honeybee Mating Optimization Algorithm to Patent Document Classification in Combination with the Support Vector Machine (SVM)," co-authors Chui-Yu Chiu and Pei-Ting Huang combine the honeybee mating optimization algorithm with SVM as a novel patent categorization approach with accurate results.

The paper, entitled "Exploring the Innovative Value of the RFID Industry" co-authored by Pei-Shu Fan, Cheng-Chin Tsao and Yi-Ching Liaw, examines a number of patent indicators to predict and explain future technological developments in the RFID industry. The research identifies the relative regional technological advantages in RFID development using relevant patent indicators and clustering analysis.

\section{References}

[1] World Intellectual Property Organization, World Intellectual Property Indicators, 2012 edition, [Online]. Available: http://www.wipo.int/ipstats/en/statistics/patents/ [Accessed: April 20, 2013]

[2] A. J. C. Trappey and P. M. Wognum, "Advanced knowledge engineering related to innovation, intellectual property and patent analysis," Advanced Engineering Informatics, Vol. 27, No. 3, pp. 315-316, 2013. doi: 10.1016/j.aei.2013.05.004
Amy J.C. Trappey is a Tsing Hua Distinguished Professor in the Department of Industrial Engineering and Engineering Management (IEEM), National Tsing Hua University (NTHU), Taiwan. She received her Ph.D. in industrial engineering from Purdue University (USA). She has taught at lowa State University and was a visiting professor and research fellow at George Mason University (USA) and Queensland University of Technology (Australia). Prof. Trappey has also served as the Dean for the College of Management at National Taipei University of Technology (2008 2011). Her research interests are in e-business, e-automation, knowledge engineering, patent analysis, and intellectual property management. Dr. Trappey is an ASME and ISEAM Fellow. 